\title{
Interactions langagières : entre perception par les apprenants et analyse de corpus par les chercheurs
}

\section{Elena Coşereanu}

\section{OpenEdition}

1 Journals

\section{Édition électronique}

URL : http://journals.openedition.org/rdlc/6362

DOI : $10.4000 /$ rdlc.6362

ISSN : 1958-5772

Éditeur

ACEDLE

\section{Référence électronique}

Elena Coşereanu, «Interactions langagières : entre perception par les apprenants et analyse de corpus par les chercheurs ", Recherches en didactique des langues et des cultures [En ligne], 5 | 2008, mis en ligne le, consulté le 04 novembre 2019. URL : http://journals.openedition.org/rdlc/6362 ; DOI : $10.4000 /$ rdlc. 6362

\section{(c) $(9) \circledast$}

Recherches en didactique des langues et des cultures is licensed under a Creative Commons AttributionNonCommercial-NoDerivatives 4.0 International License 


\title{
Interactions langagières : entre perception par les apprenants et analyse de corpus par les chercheurs
}

\author{
Elena Coşereanu \\ Université de Technologie de Compiègne (COSTECH - EA 2223), France
}

\section{Résumé}

De nombreuses études sur la tâche d'apprentissage réalisées en communication synchrone textuelle (clavardage) et orale (en face-à-face) ont montré que les tâches d'écart d'information (ex : puzzle et prise de décision) pouvaient contribuer à l'acquisition d'une langue seconde. Dans l'étude expérimentale que nous présentons ici, nous avons voulu d'une part analyser les interactions langagières observables dans différentes tâches d'écart d'information réalisées en binôme par des apprenants en français langue étrangère (FLE), et d'autre part interroger les participants sur leur perception de l'effet de ce genre de tâche en tant que facteur contribuant à leur apprentissage.

Les apprenants ont réalisé trois types de tâche d'apprentissage dans les deux modalités de communication synchrone étudiées (clavardage et oral) : 1) trouver les différences entre deux images apparemment identiques ; 2) résoudre une énigme ; 3) raconter une scène d'un film venant d'être visualisé. Nous avons complété notre recueil de données avec deux questionnaires destinés à évaluer: 1) le niveau des participants en FLE;2) leur attitude personnelle à l'égard de ces activités (notamment leur opinion quant à l'utilité des tâches proposées et quant à leur impact motivationnel).

Nous présenterons le cadre théorique de cette étude et ses résultats généraux. Nous aborderons également la question des difficultés méthodologiques qui se posent quand on cherche à déterminer si les tâches ont effectivement déclenché le processus d'apprentissage ou non, et nous discuterons brièvement à ce titre des insuffisances d'une démarche utilisant des pré- et des post-tests.

\section{Mots-clés}

interaction, communication synchrone, tours de négociation, rétroaction corrective 


\begin{abstract}
Several studies on Acquisition Tasks in text (chat) or face-to-face synchronous communication have indicated that information gap tasks (for example jigsaw or decision making tasks) could contribute to second language acquisition. The aim of the present experimental study was to analyse the communicative interactions in different information gap tasks carried out by pairs of learners in a French as a foreign language classes, and to study the participants' perception of the effect of those tasks as a factor contributing to their language acquisition.

The learners performed three types of task in the two modalities of synchronous communication studied (chat and face-to-face) : 1) finding differences between two pictures that seem identical; 2) solving an enigma; 3) relating a scene of a movie while watching. We completed our data collection with two questionnaires aimed at evaluating: 1) the participants' level of French; 2) their personal attitude toward the tasks (in particular their opinion on the usefulness of the tasks and their motivational impact).

We will present the theoretical background for this study and our overall results. We will also raise the question of the methodological difficulties that are faced when trying to determine if tasks have effectively triggered the learning process, and briefly discuss the insufficiencies of an approach that uses pre- and post-tests.
\end{abstract}

\title{
Keywords
}

interaction, synchronous communication, negotiating interaction, corrective feedback 


\section{Le rôle des tâches d'apprentissage dans l'acquisition des langues secondes}

\subsection{Interactions négociées et tâches d'apprentissage}

Les interactions réalisées par les apprenants travaillant en binôme de locuteurs non-natifs (LNN-LNN) ou de locuteurs natifs et de locuteurs non-natifs (LN-LNN) lors d'une communication synchrone représentent un sujet important pour l'acquisition des langues secondes. Les tâches d'apprentissage constituent un moyen de produire l'interaction, qui est un vecteur majeur d'apprentissage d'une nouvelle langue (Prabhu, 1987; Ellis, 2003; Kazeroni, 2004).

Il est cependant clair que toutes les tâches qui génèrent des interactions ne donnent pas lieu à l'apprentissage et que toute interaction ne facilite pas l'acquisition. La question se pose de savoir si certaines tâches sont plus appropriées que d'autres pour générer des interactions débouchant sur l'apprentissage.

Or, les recherches en acquisition des langues secondes ont montré que les interactions dites "négociées" étaient un type d'interaction favorisant de manière insigne l'apprentissage. L'interaction négociée se définit comme une interaction où un ou plusieurs signaux d'incompréhension donnent lieu, par l'intermédiaire d'une rétroaction corrective, à une résolution des énoncés problématiques. L'interaction est dans ce cas bénéfique dans la mesure où l'apprenant exposé à la rétroaction corrective peut prendre conscience des écarts entre son interlangue et la langue-cible, et restructurer ou compléter son interlangue afin de les réduire (Gass, 1997 ; Long, 1996 ; Pica, 1994 ; Ellis, 2003). De ce point de vue, la question de la pertinence des tâches pour l'apprentissage revient à déterminer quel type de tâche encourage la négociation (de la forme / des formes comme du sens).

Pica (1988 : 54-55), en utilisant le modèle de l'interaction négociée de Gass \& Varonis (1985), a proposé de distinguer, parmi les négociations entre les LN-LNN, les interactions négociées avec un seul signal (où un seul signal manifeste une difficulté de compréhension) des interactions négociées étendues (plusieurs signaux de non compréhension), insistant ainsi sur l'importance de la différence de longueur des négociations. D'un point de vue formel, l'interaction avec un seul signal comprend : l'énoncé du déclencheur, le signal de la non compréhension, la réponse au signal, la résolution. Dans les interactions négociées étendues, les négociations sont plus longues et chaque interlocuteur signale la non-compréhension au 
moins une fois. Ce type de négociation inclut les séquences suivantes: l'énoncé du déclencheur, le signal de la non compréhension, la réponse au signal, le signal du manque (de l'absence) de la compréhension, la réponse au signal, la résolution, les signaux de la noncompréhension continus ou abandon de la négociation. De ces deux types d'interaction, il semble que les interactions négociées étendues soient plus propices à générer l'apprentissage. En effet, plus il y a de négociations, plus il y a d'opportunités pour une restructuration de l'interlangue, donc plus de chances pour la création d'un discours cohérent en langue cible. Ces interactions étendues pourraient être l'environnement requis pour remarquer l'écart ou la non-correspondance (mismatch) entre l'interlangue de l'apprenant et la L2 (Gass et al. 1998 ; Braidi, 2002), se focaliser sur la forme (Long, 1996), et faciliter le développement de la L2. Si on accepte que l'interaction négociée facilite le développement de la L2 (Gass et al. 1988; Long, 1996 ; Pica, 1994), il semble ainsi qu'il faille accorder une plus grande attention aux interactions étendues, où les apprenants manifestent plus de difficultés à modifier leur output que dans des interactions simples.

De façon générale, il est également admis que la rétroaction corrective peut faciliter le développement de la L2 (Long, 1996 ; Tatawy, 2003). Lightbown et Spada (1999) définissent la rétroaction corrective comme suit :

Any indication to the learners that their use of target language is incorrect. This includes various responses that the learners receive. When a language learner says, "He go to school every day", corrective feedback can be explicit, for example, "no, you should say goes, not go" or implicit "yes he goes to school every day", and may or may not include metalinguistic information, for example, "Don't forget to make the verb agree with the subject". (Lightbown et Spada, 1999 : 171-172)

Par ailleurs, dans la mesure où la rétroaction corrective représente la structure communicationnelle sur laquelle l'interaction négociée repose, si cette dernière facilite bel et bien le développement de la L2, la capacité d'une tâche d'apprentissage à générer des rétroactions peut être prise comme un indicateur de sa valeur pour l'acquisition. 


\subsection{Rôle de la modalité communicationnelle pour l'acquisition}

Dans la mesure où les tâches d'apprentissage peuvent se réaliser suivant différentes modalités communicationnelles, typiquement l'écrit ou l'oral, la question se pose également de savoir si une modalité est plus adaptée qu'une autre pour susciter l'acquisition.

Les études qui ont comparé le clavardage à la conversation en face-à-face ont montré que le clavardage pouvait constituer une passerelle intéressante entre la compétence d'expression écrite et celle d'expression orale (Chun, 1994), qu'un plus grand nombre d'échanges étudiantétudiant avaient lieu lors de sessions sur ordinateur que lors de sessions en face-à-face avec un enseignant (Kern, 1995), que la participation des étudiants augmentait et était plus également répartie dans les échanges électroniques que dans ceux qui se déroulent en face-àface (Warschauer, 1996), et qu'il y avait une amélioration de la compétence orale des participants (après deux mois d'activités de clavardage dans l'étude de Negretti, 1999). Des logiciels tels $Y$-Talk permettent aux apprenants de visionner et d'interrompre (comme lors d'une discussion orale) l'élaboration des messages des autres participants à mesure que ceuxci les écrivent. Ce procédé est différent des outils de clavardage courants où les participants ne peuvent voir que la version finale envoyée par les interlocuteurs (Pellettieri, 2000). On a constaté une grande part d'autocorrection lors des conversations par clavardage, comme l'indiquent l'utilisation de la touche d'effacement et la réparation des erreurs typographiques, orthographiques et morphologiques (notamment les erreurs d'accord), et on a observé que la majorité des corrections explicites et implicites conduisaient à des incorporations des formes cibles et perturbaient souvent la dynamique de la conversation (Tudini, 2003 : 66). Il semble également que la plupart des négociations menées par clavardage en binôme soient initiées par des confusions lexicales plutôt que morphologiques ou syntaxiques.

On peut également remarquer que les réparations ${ }^{1}$ semblent surtout être une caractéristique du discours oral, qui peut être initiée par une rétroaction implicite ou explicite de la part de l'interlocuteur ou qui peut être de l'initiative du locuteur lui-même (autocorrection). Une attention particulière a été apportée aux réparations, ainsi qu'à toute forme d'incorporation des formes cibles qui résulterait de celles-ci, l'incorporation étant considérée par les

\footnotetext{
${ }^{1}$ Long (2005) définit la réparation (recast) comme "une reformulation de tout ou d'une partie d'un énoncé immédiatement précédent d'un apprenant, dans lequel un ou plusieurs items erronés (lexical, grammatical, etc.) sont remplacés par les formes correctes correspondantes du langage cible, et où, à travers l'échange, la concentration des interlocuteurs est sur le sens." Voir également Long (1996:434).
} 
chercheurs acquisitionnistes comme un indicateur majeur de la négociation du sens, et de ce fait d'un éventuel développement de l'interlangue (Tudini, 2003 : 71).

\section{Approche expérimentale}

Le but de l'étude expérimentale que nous avons réalisée était de comparer différents types de tâches d'écart d'informations ${ }^{2}$ réalisées en binôme, par clavardage ou à l'oral, afin de déterminer si celles-ci présentaient une potentialité différente à générer des types d'interaction langagière susceptibles de favoriser le processus d'acquisition (tours de négociations, rétroaction, réparation), et le cas échéant, quels caractères de ces tâches pourrait expliquer ces différences. Nous avons également essayé d'observer de quelle manière les apprenants avaient perçu ces différentes tâches d'apprentissage, en particulier leur difficulté.

C'est un enjeu important pour la didactique des langues de déterminer les caractéristiques des tâches d'apprentissage qui rendent celles-ci efficaces pour l'acquisition des langues secondes, et sous quelle modalité de communication cette efficacité est maximisée.

Pour cette étude, nous avons pris pour base les hypothèses suivantes, généralement accréditées par la littérature de spécialité : 1) l'exposition de l'apprenant à la rétroaction corrective peut favoriser l'amélioration de ses connaissances ; 2) l'incorporation peut être interprétée, dans certaines circonstances, comme un indicateur d'une restructuration en cours de l'interlangue, c'est-à-dire comme l'amorce d'une appropriation future.

\subsection{Méthode et participants}

Nous avons réalisé cette étude expérimentale avec 12 apprenants volontaires de FLE (de 8 nationalités, âgés de 18 à 24 ans), organisés en 6 binômes, au Centre de Langues de l'Université de Technologie de Compiègne pendant le mois d'août $2007^{3}$. Le binôme semble la configuration la plus adaptée pour des sessions de communication assistée par ordinateur pour des apprenants de langues (Pelletieri, 2000 ; Blake, 2000 ; Tudini, 2003).

Chaque binôme a réalisé trois types de tâches, dans les deux conditions de communication étudiées (clavardage et oral en face-à-face). Nous avons finalisé notre recueil de données avec

\footnotetext{
${ }^{2}$ Une tâche d'écart d'information est une tâche où les participants ne possèdent pas les mêmes informations et dont la résolution nécessite l'utilisation et la mise en regard (par ex. dans une comparaison) des informations de chacun (cf. Ellis, 2003).

${ }^{3}$ Nous avions initialement 11 binômes, mais 2 binômes ont finalement abandonné l'expérience, et 3 binômes n'ont réalisé les tâches qu'à l'oral ; comme nous voulions comparer les modalités de communication orale et écrite, nous les avons écartés.
} 
deux questionnaires destinés à évaluer : 1) le niveau des participants en FLE ; 2) leur attitude personnelle à l'égard de ces tâches d'apprentissage (notamment leur opinion quant à l'utilité des tâches proposées et leur difficulté $)^{4}$. Les questionnaires ont fait apparaître que les participants avaient un niveau en FLE variable (niveau compris entre A1 et B2, d'après le CECRL du Conseil de l'Europe publié en 2000).

Les interactions orales et les entretiens oraux ont été transcrits et vérifiés ${ }^{5}$.

\subsection{Tâches d'apprentissage utilisées et consignes}

Nous avons proposé aux étudiants participant à notre étude trois tâches d'apprentissage du type écart d'informations : 1) raconter une scène d'un film de comédie français (visionnée avec ou sans son);2) trouver les différences entre deux images apparemment identiques (d'après le modèle déjà utilisé par Mackey et Gass, 2005) ; 3) résoudre une énigme à partir d'une série d'énoncés textuels.

\subsubsection{Tâche 1 : raconter une scène d'un film}

Chaque binôme se voit présenté une séquence d'un film français de comédie, l'un des deux étudiants visionnant la scène avec le son (en utilisant des écouteurs), alors que l'autre ne bénéficie que de l'image (pas d'écouteurs). Après avoir visionné la séquence, chaque étudiant du binôme doit raconter à son partenaire avec le maximum de détails le contenu de la scène. Pour le clavardage, la scène visionnée était un extrait du film "La Chêvre", avec Gérard Depardieu et Pierre Richard, où le personnage joué par P. Richard a une altercation avec un autre personnage au sujet d'un chariot à l'aéroport. La scène dure 2'30 et comprend beaucoup de vocabulaire argotique, ce qui peut représenter une difficulté pour les apprenants. Pour l'activité orale, il s'agissait du film "L'aile ou la cuisse", avec Louis De Funès (séquence de 4’30), les étudiants inversant cette fois leurs conditions de visionnement : celui ayant bénéficié du son pour la séquence du premier film regardait cette fois le film sans son, et inversement.

\footnotetext{
${ }^{4}$ Le premier est un questionnaire écrit d'autoévaluation qui contient 3 parties (sur les stratégies d'apprentissage, le niveau en FLE et l'habitude du clavardage et de l'oral). Le deuxième est un entretien oral semi-dirigé qui contient 12 questions sur les tâches d'apprentissage réalisées, les erreurs grammaticales, lexicales ou de prononciation des étudiants et les difficultés pour parfaire leurs propres connaissances en FLE.

${ }^{5}$ Les communications par clavardage ont été enregistrées au format $h t m l$ et les communications orales au format mp3.
} 


\subsubsection{Tâche 2 : trouver les différences entre deux images apparemment}

\section{identiques}

Pour la tâche réalisée par clavardage, consistant à trouver les différences entre deux images apparemment identiques, nous avons repris les images utilisées par Mackey et Gass (2005). Chaque participant a sous les yeux une image (représentant une cuisine aménagée, cf. figure 1) et doit décrire à son partenaire son contenu afin qu'ils puissent déterminer si leurs images présentent des différences et lesquelles. Le but est de trouver le maximum de différences entre les images, sachant qu'il y avait au total 10 différences.

Pour l'activité orale, nous avons utilisé les deux images représentées sur la figure 2 (8 différences à trouver). La consigne est la même que pour le clavardage.
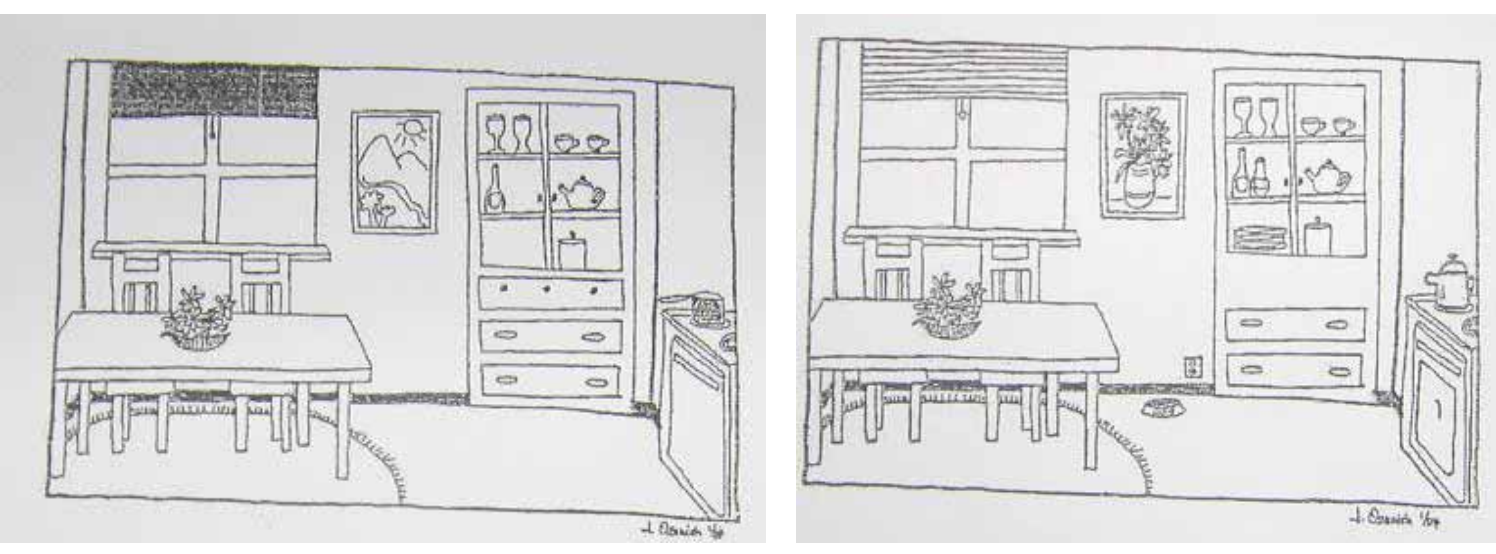

Figure 1 - Images "Cuisine" utilisées dans la tâche n² (Clavardage).
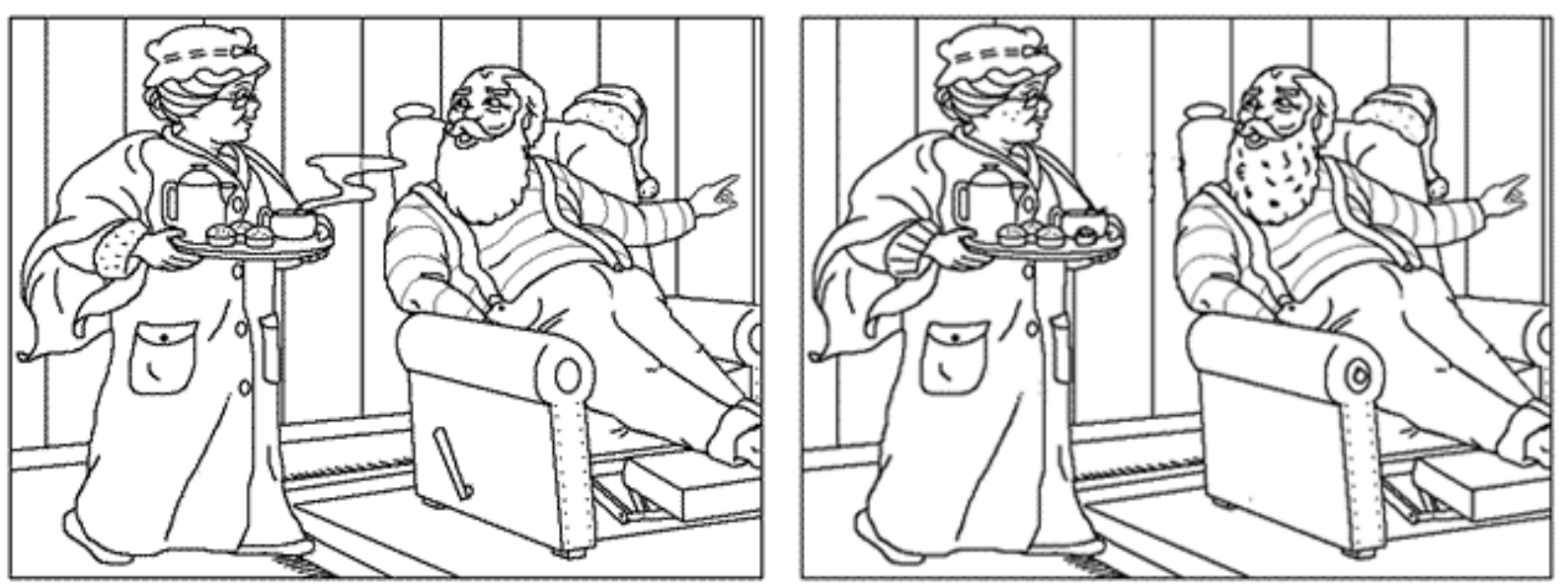

Figure 2 - Images "Père Noël" utilisées dans la tâche n² (Oral). 


\subsubsection{Tâche 3 : résoudre une énigme}

Pour la troisième tâche, nous avons choisi une énigme comportant six indices sous forme d'énoncés. Chaque étudiant se voit attribué trois des six indices et doit expliquer à son binôme le contenu de ses énoncés, de manière à ce qu'ils puissent résoudre ensemble l'énigme. Pour le clavardage, l'énigme était la suivante :

"Messieurs Leblanc, Lenoir et Leroux sont des enseignants. Il y a cinq sujets à enseigner : anglais, français, maths, histoire, géographie.

Indices : 1) Monsieur Lenoir ne sait pas ce qu'est un angle ; 2) Monsieur Leblanc est le seul à savoir où sont les montagnes Rocheuses; 3) chacun enseigne trois matières ; 4) aucune matière n'est enseignée par 3 personnes; 5) certaines matières sont enseignées par 2 personnes ; 6) Monsieur Leroux est bilingue.

Question : Qui enseigne quelles matières ?".

Pour l'oral, nous avons choisi une énigme ("Les cartes de poker") consistant à identifier trois cartes de poker dont on ne voit pas les faces à partir de quatre énoncés fournissant des indices. Le degré de difficulté de cette tâche était plus grand que pour les autres, notamment puisque sa résolution présupposait la mobilisation d'une plus grande attention et une connaissance de la logique :

"Trois cartes sont placées sur une table de poker. On ne voit pas leurs faces.

Indices : 1) à gauche de la reine, il y a le valet ; 2) à la gauche d'un pique, il y a un carreau ; 3) à la gauche d'un coeur, il y a un valet ; 4) à la droite d'un roi, il y a un pique.

Question : identifiez les cartes."

\section{Analyse et interprétation des données}

Afin de déterminer si ces différentes tâches et les deux modalités de communication étudiées étaient plus ou moins adaptées pour la génération de types d'interactions langagières susceptibles de favoriser l'apprentissage, nous avons analysé les communications réalisées à l'aide d'une grille des types d'interactions langagières, et avons comparé leur nombre par tâche d'apprentissage et par modalité. Nous ne rapportons ici que les résultats pour les routines de négociation, les rétroactions correctives et l'incorporation, types d'interactions qui semblent jouer le rôle le plus important pour le déclenchement du processus de restructuration des acquis et l'apprentissage. 
Concernant les routines de négociation, l'analyse statistique des données ne permet pas de déterminer s'il y a un effet de la tâche ou de la modalité sur le nombre de routines de négociation générées dans les communications (ANOVA p=0,1439). On observe seulement une différence statistiquement significative entre le nombre de tours de négociation générés dans les deux modalités (ANOVA $\mathrm{F}(1,5)=7,8344, \mathrm{p}=0,0380$ ) : en moyenne 11,17 tours de négociation par communication en clavardage, et 6,11 à l'oral. Ce résultat pourrait indiquer que les routines de négociation sont globalement plus longues en clavardage qu'à l'oral. Ce phénomène pourrait s'expliquer par l'absence d'indices paralinguistiques en modalité de communication clavardage, qui doit être palliée par une négociation plus longue : plus de tours de paroles sont nécessaires pour arriver à un terrain d'entente.

Concernant maintenant le nombre de rétroactions correctives ${ }^{6}$ (Cf. figure 3), les résultats indiquent que la tâche "image" est la plus efficace pour générer des rétroactions correctives, suivie par la tâche "film" (ANOVA F(2,10)=13,6164, p=0,0014). En clavardage, la tâche "image" génère plus de rétroactions correctives que les deux autres tâches. Cependant à l'oral, elle génère plus de rétroactions correctives que la tâche "énigme" mais pas que la tâche "film". Par ailleurs, la tâche "film" génère plus de rétroactions correctives que la tâche "énigme" en clavardage comme à l'oral (ANOVA $F(1,5)=10,0000, p=0,0250$ ). On observe également que la modalité clavardage augmente la production de rétroactions correctives pour la tâche "image" (ANOVA F(1,5)=62,8151, p=0,0005).

On retrouve les résultats de Gass, Mackey et Ross-Feldman (2005: 596) ${ }^{7}$ qui ont observé qu'une tâche de type "image" générait plus de rétroactions correctives que les autres tâches de communication comparées. Toutefois, les auteurs n'avaient pour leur part comparé la tâche "image" à d'autres tâches qu'en modalité orale. Nos résultats, quant à eux, tendent à indiquer que la tâche "image" génère plus de rétroactions correctives (notamment de réparations) que les autres tâches uniquement pour la modalité clavardage (voir figure 3).

\footnotetext{
${ }^{6}$ Pour les valeurs présentées, nous avons additionné les rétroactions correctives implicites (réparations), explicites, incorrectes, et les autocorrections.

${ }^{7}$ Voir également l'étude de Varonis et Gass (1985).
} 


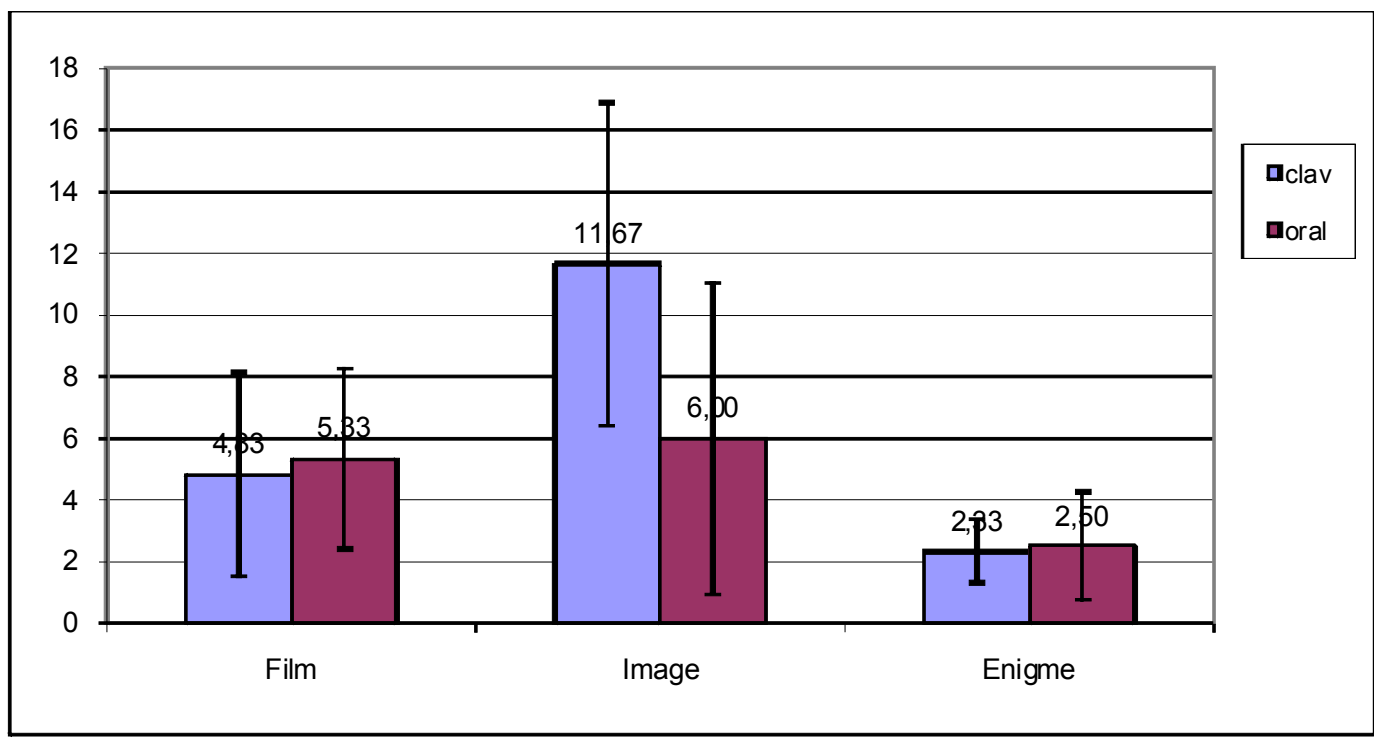

Figure 3 - Nombre moyen de rétroactions correctives par tâche et modalité.

Cependant, comme on peut le voir sur le graphe de la figure 4, on observe également un nombre moyen de tours de parole beaucoup plus important pour la tâche "image" que pour la tâche "film" et la tâche "énigme" (ANOVA $F(2,10)=6,9985, \mathrm{p}=0,0126)$, ce qui indique que les communications de la tâche "image" étaient globalement plus étendues que pour les deux autres tâches d'apprentissage.

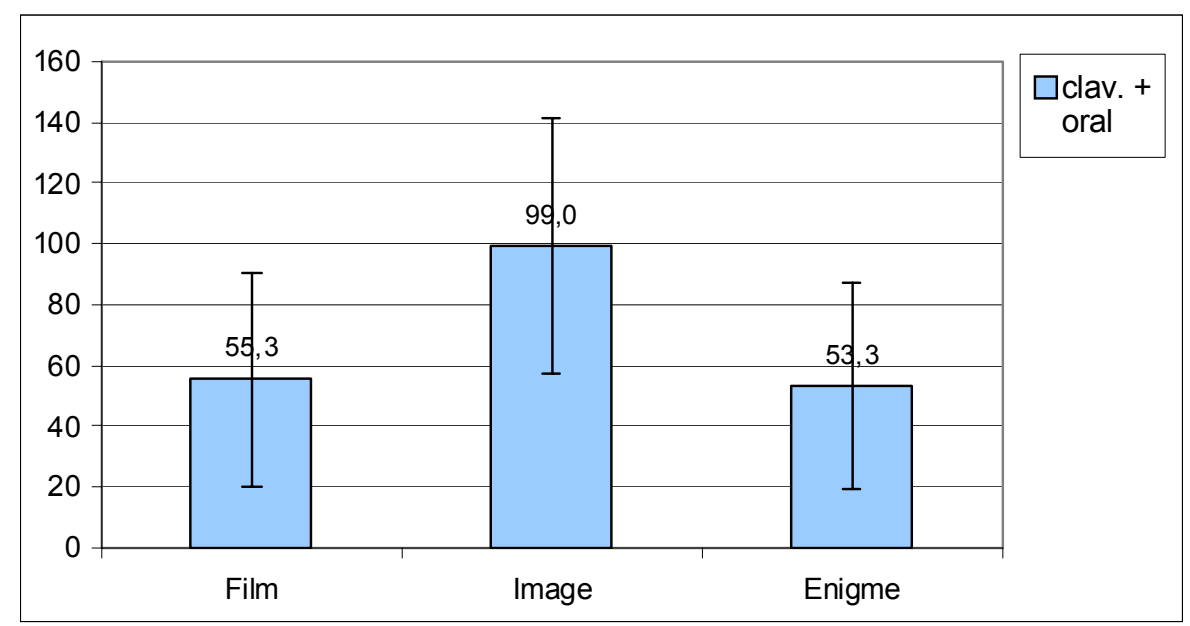

Figure 4 - Nombre moyen de tours de parole par tâche.

Afin de déterminer si cette différence dans le nombre de tours de parole pourrait en partie expliquer la différence observée entre le nombre de rétroactions correctives générées dans les différentes tâches, nous avons rapporté le nombre de rétroactions correctives observé pour chaque tâche dans les deux modalités au nombre de tours de parole correspondant (Cf. Figure 5). Or, on observe qu'une fois rapporté au nombre moyen de tours de parole, le 
nombre moyen de rétroactions correctives générées dans la modalité clavardage est globalement équivalent pour la tâche "film" et la tâche "image" et est plus important que pour la tâche "énigme". À l'oral, le nombre de rétroactions correctives est plus important pour la tâche "film" que pour les tâches "image" et "énigme" où il est globalement équivalent. Ces résultats pourraient donc indiquer que la tâche "image" a permis la production d'un nombre de rétroactions correctives plus important parce qu'elle a donné lieu à des interactions plus longues (plus de tours de parole), et non en raison d'une fréquence d'apparition des rétroactions correctives plus importante.

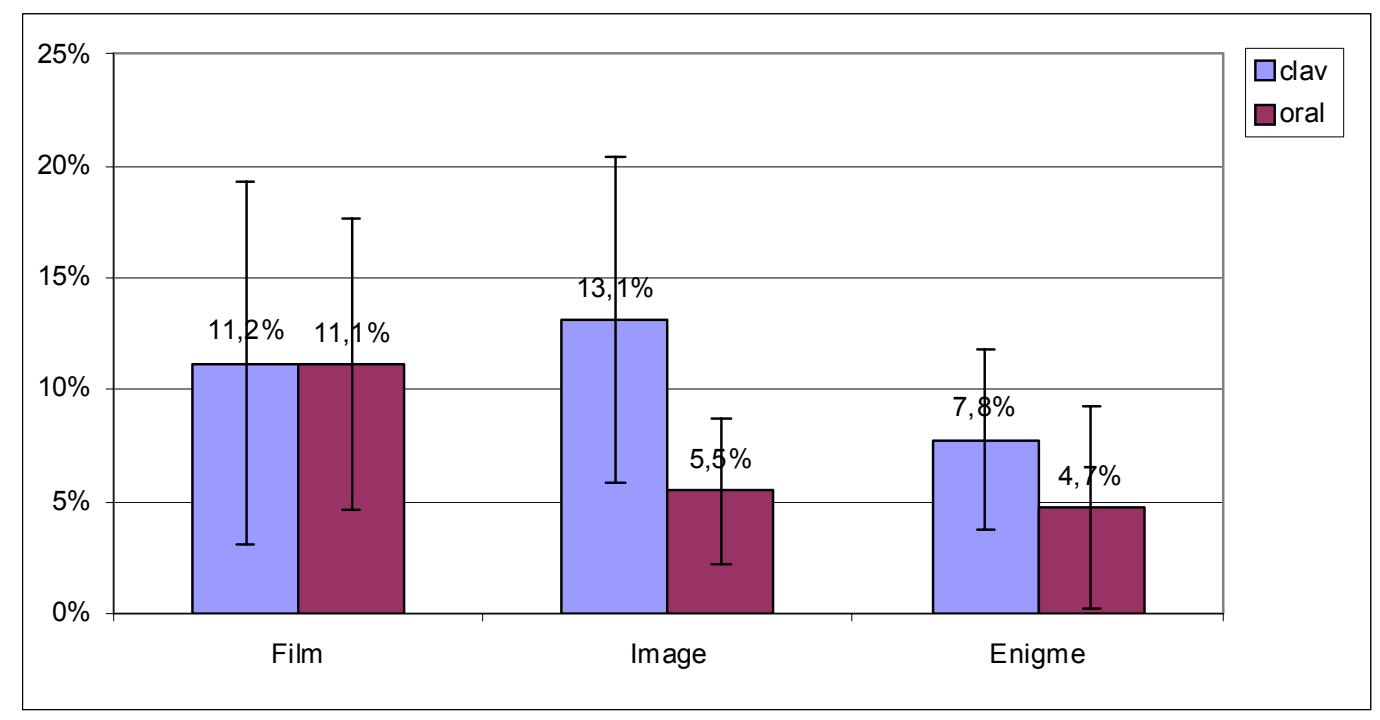

Figure 5 - Nombre moyen de rétroactions correctives par tâche et modalité rapporté au nombre moyen de tours de parole.

Les questionnaires remplis par les participants à la fin de l'expérience, destinés à évaluer leur perception des différentes tâches, peuvent en partie nous aider à expliquer pourquoi la tâche "image" a généralement donné lieu à un nombre plus important de tours de parole que les deux autres tâches, et donc pourquoi, cet élément pris en compte, la tâche "image" s'avérait finalement être celle donnant lieu au nombre le plus important de rétroactions correctives (notamment en clavardage). En particulier, nous avons interrogé les participants sur leur perception de la difficulté des différentes tâches réalisées (cf. figure 6). 


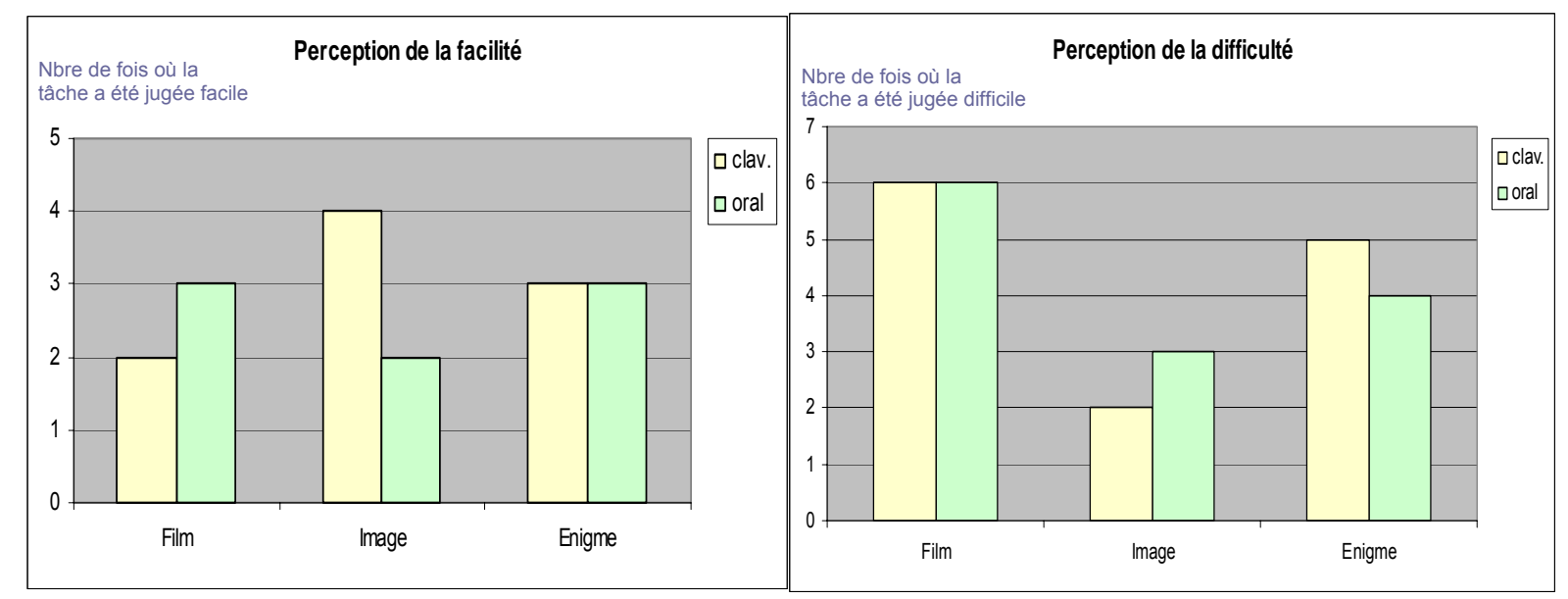

Figure 6 - Perception de la facilite / difficulté de la tâche.

Or, comme on peut le voir, la tâche "image" est généralement estimée la plus facile (surtout en clavardage), alors que la tâche "film" est la tâche estimée la plus difficile aussi bien en clavardage qu'à l'oral. Ces résultats nous autorisent à faire l'hypothèse que le nombre de tours de parole plus important observé dans la tâche "image" n'est pas dû à une difficulté accrue de la tâche (cette difficulté accrue aurait pu expliquer que les communications soient plus longues, la tâche exigeant plus de temps pour sa résolution), mais peut-être à une motivation plus grande, et en fait à un bon dosage entre la difficulté de la tâche et son attractivité. Que la tâche présente une plus grande attractivité expliquerait en partie que les participants se soient portés au jeu d'une communication plus longue (puisque les participants pouvaient à tout moment décider d'arrêter la communication). Nous proposerons plus loin plusieurs pistes pour expliquer cette différence de motivation entre les différentes tâches, et de là formuler une série de propositions visant à maximiser la portée motivationnelle des tâches utilisées pour l'enseignement / apprentissage.

Concernant finalement l'impact de la modalité de communication, les résultats indiquent un effet de la modalité sur le nombre moyen d'incorporations : il y a en moyenne plus d'incorporations à l'oral que par clavardage (ANOVA $F(1,5)=9,4944, p=0,0274$ ) (Cf. figure 7). Il n'y a en revanche pas d'effet de la tâche. Les données indiquent également un effet important de la modalité sur le nombre de rétroactions correctives, plus nombreuses en clavardage qu'à l'oral (ANOVA $\mathrm{F}(1,5)=53,5714, \mathrm{p}=0,0007)$. La modalité clavardage semble donc favoriser la production de rétroactions correctives mais pas la production d'incorporations. Par ailleurs, nos données ne permettent pas d'affirmer l'existence d'une relation entre le nombre de rétroactions correctives et le nombre d'incorporations. 

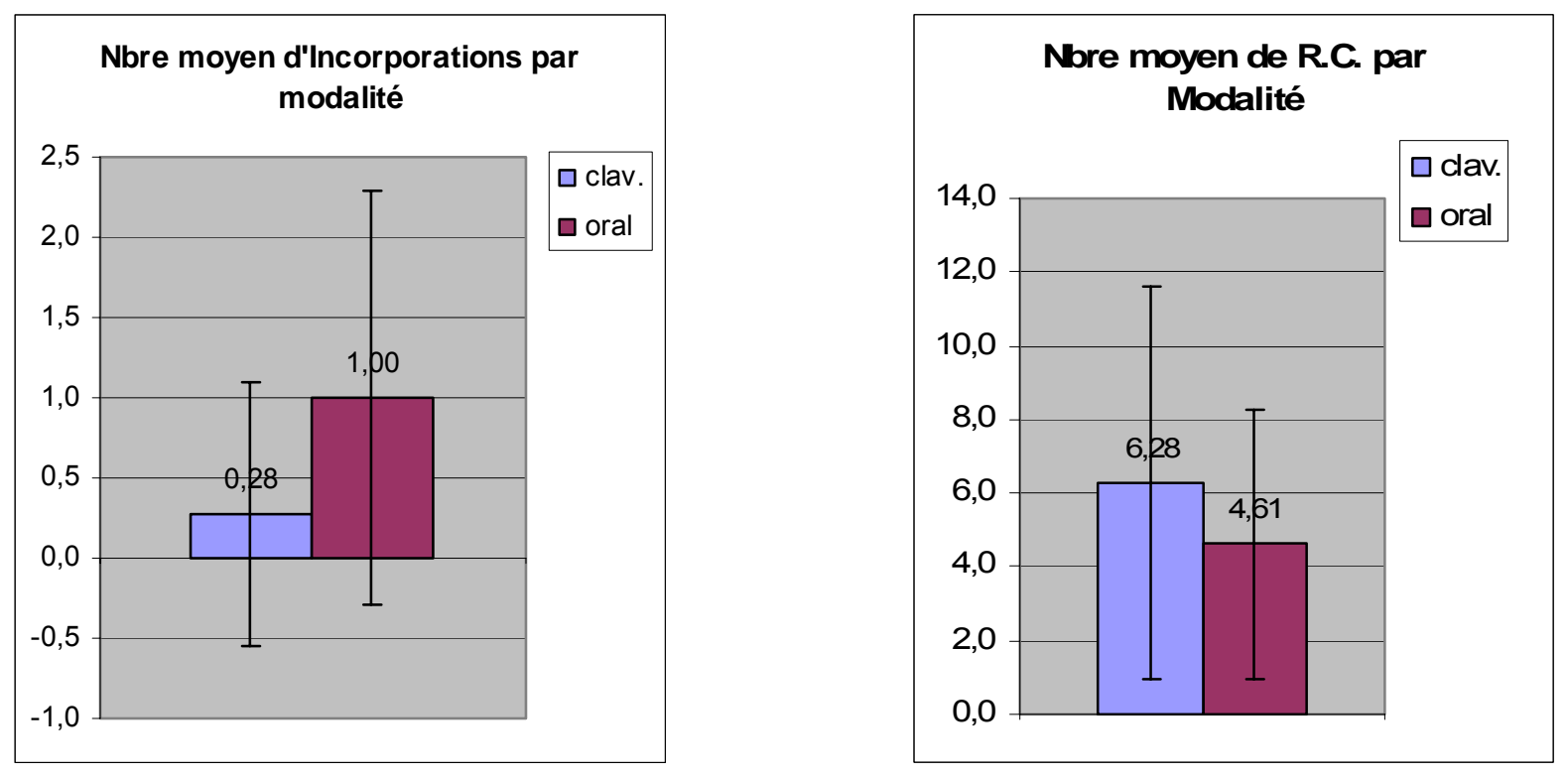

Figure 7 - Effet de la modalité sur le nombre d'incorporation et de rétroactions correctives.

On remarquera que la différence de temporalité de l'interaction entre l'écrit et l'oral (plus de temps de réflexion à l'écrit, persistance visuelle du message) pourrait expliquer qu'un plus grand nombre de rétroactions correctives soient produits en modalité clavardage.

\section{Exemples de types d'interactions langagières extraits du corpus}

\subsection{Rétroaction corrective explicite}

Dans ce type de rétroaction, l'erreur est corrigée de manière explicite (cf. supra, la définition de Lightbown et Spada, 1999). Le correcteur signale au locuteur fautif qu'il a commis une erreur. Toutefois, il faut préciser que ce type de rétroaction ne comporte pas nécessairement d'informations métalinguistiques (c'est-à-dire d'informations portant sur les règles linguistiques). La rétroaction corrective explicite peut être de nature lexicale, grammaticale ou discursive. L'exemple suivant illustre une rétroaction explicite lexicale :

\begin{tabular}{|l|l|l|l|}
\hline SA & $23 / 08 / 2007$ & $17: 50: 34$ & il est cuisine ????? \\
\hline MM & $23 / 08 / 2007$ & $17: 50: 52$ & $\begin{array}{l}\text { non, cuisine c'est le lieu [rétroaction corrective explicite } \\
\text { lexicale] }\end{array}$ \\
\hline
\end{tabular}




\subsection{Rétroaction corrective implicite ou réparation (recast)}

La rétroaction corrective implicite ou réparation (recast) corrige l'erreur commise de manière implicite, c'est-à-dire sans que le correcteur signale au locuteur qu'il a commis une erreur, et donc sans interrompre le flux de la communication. La réparation peut être de nature lexicale, grammaticale ou discursive (Long, 2005). Les réparations sont fondamentalement ambiguës (Tarone et al., 2003), car les répétitions des énoncés de l'interlocuteur peuvent être interprétées comme des énoncés où l'attention est focalisée soit sur le sens, soit sur la forme, soit sur les deux. Voici des exemples de réparations fréquentes dans notre étude :

\section{Réparation lexicale}

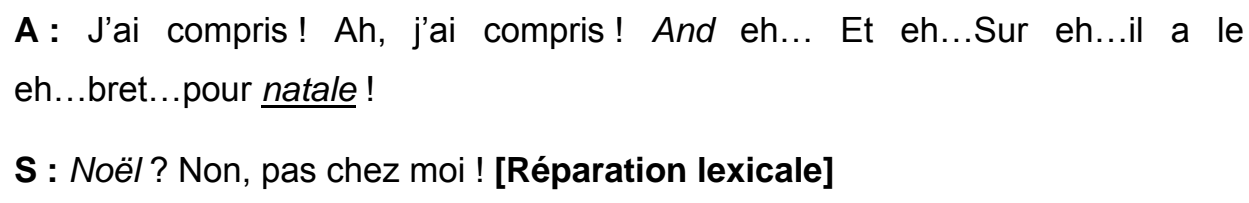

Dans cet exemple, l'étudiant italien ne connaît pas le mot "Noël" en français et utilise la forme telle qu'elle existe dans sa langue maternelle ("natale"). La correction de la part de son interlocuteur (Néerlandais) se produit immédiatement et implicitement.

\section{Réparation grammaticale}

$$
\begin{aligned}
& \text { S : et un table } \\
& \text { M: il y a une table aussi ? [Réparation grammaticale] }
\end{aligned}
$$

\section{Réparation phonétique}

Lors des communications en face-à-face, les étudiants font des erreurs de prononciation, corrigées le plus souvent de manière implicite :

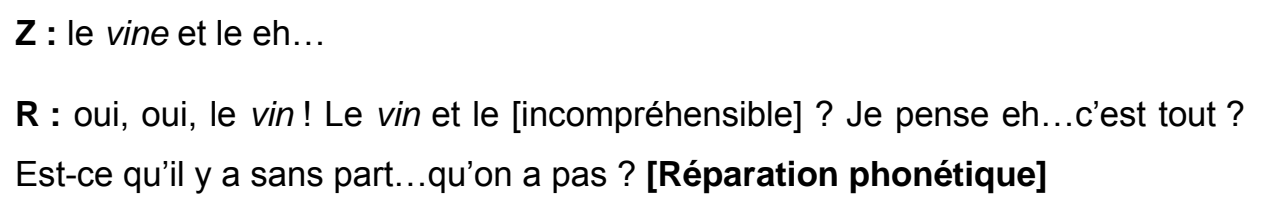

\section{Réparation incorrecte}

De même, lorsque la rétroaction corrective est incorrecte, c'est-à-dire quand elle ne contient pas une évidence positive, nous parlons de réparation incorrecte, comme dans l'exemple suivant portant sur l'utilisation du verbe jouer (aux cartes) :

$$
\text { F : Il joue, il a joué cartes avec son ami ! Avec ....avec sa ... }
$$


C : ah, jouer les cartes! Ah, oui ! Ah, il est jouer, et Monsieur, anglais ! Et c'est...Qu'est-ce que c'est? II or...Mais il n'a pas mangé ! [Rétroaction implicite incorrecte $I$ réparation grammaticale incorrecte]

\section{Autocorrection (rétroaction autocorrective)}

L'autocorrection (catégorie à part de la rétroaction corrective) est produite par le locuteur même qui fait l'erreur. Le locuteur reformule l'énoncé comportant l'erreur qu'il a commise, mais cette fois-ci avec la forme correcte (grammaticale, lexicale ou sémantique / discursive).

H : Oui ! Une femme et une homme ! un homme ! J'ai...j'ai le Père Noël!

[Autocorrection grammaticale]

\section{Rétroaction non saisie (no feedback)}

Lorsqu'une erreur est produite sans être saisie / corrigée par l'autre membre du binôme, nous parlons de rétroaction non saisie.

$$
\begin{aligned}
& \text { C : Je vous dit les raisons } \\
& \text { H : Oui [rétroaction non saisie] }
\end{aligned}
$$

\subsubsection{Incorporation (uptake)}

L'incorporation consiste, pour un apprenant ayant commis une erreur que son interlocuteur vient de réparer, à répéter la forme corrigée dans un nouvel énoncé. Par définition, l'incorporation (uptake) (Lyster and Ranta, 1997 : 49) suit immédiatement une réparation. Elle peut être immédiate :

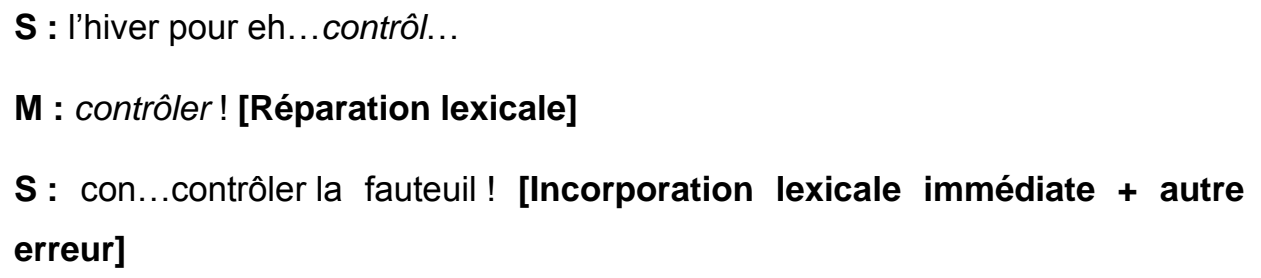

Mais il y a aussi des cas où l'incorporation apparaît quelques tours après la réparation ou même plus tard dans la conversation. L'incorporation est dans ce cas dite différée :

$\mathbf{R}$ : comme ça ! Oui, tombé ! Le bruit tombé !

Z : le bruit est tombé ! C'est ça et eh... cet homme est tombé ! Pourquoi ? rétroaction corrective explicite grammaticale

$(\ldots)$ 
$\mathbf{R}$ : cet homme... cet homme il fit ça il est tombé... je ne sais pas...

[Incorporation grammaticale différée]

\section{Discussion}

On peut, pour expliquer les différences communicationnelles (en particulier nombre de tours de parole et de rétroactions correctives) observées entre les différentes tâches d'apprentissage et modalités, proposer plusieurs hypothèses.

Tout d'abord, concernant ce que nous proposons ici d'appeler la dimension "collaborative" : la tâche "image" semble être celle des trois tâches qui exige le degré de collaboration le plus important pour être résolue : dans la mesure où chacun des deux partenaires a besoin que l'autre lui décrive précisément le contenu de son image, il ne peut opérer seul. La tâche "film" n'a pas véritablement d'enjeu, puisqu'il faut juste raconter ce qu'on a compris du passage du film visionné : il n'y a pas de réel problème à résoudre, mais il est cependant nécessaire d'arriver à un terrain d'entente sur le sens de la séquence visionnée. Enfin, la tâche "énigme" semble être celle dont le degré de collaboration exigé est le plus faible, puisque rien n'interdit aux participants de livrer d'emblée leurs indices à leur partenaire. Il était ainsi fréquent de voir les participants livrer "en bloc" dès le début de l'interaction leurs énoncés.

Concernant les différences de contenu entre les différentes tâches, on peut remarquer que la tâche "image" contient des éléments iconographiques et non pas du texte, alors que la tâche "énigme" possède a contrario un contenu uniquement textuel. La tâche "film" étant de ce point de vue à mi-chemin entre les deux. Il faut également remarquer que la tâche "image" exige la traduction de l'information iconographique en une information langagière (textuelle ou orale selon la modalité de la tâche) pour sa transmissibilité.

Ces différences dans le degré de collaboration et le contenu des différentes tâches pourraient expliquer les différences observées dans le nombre et la fréquence des types d'interaction langagière que nous avons étudiés. En particulier, le fait que la tâche "film" et la tâche "image" présentaient une fréquence de génération de rétroactions correctives plus importante par rapport à la tâche "énigme" semble clairement pouvoir s'expliquer par ces caractères. Le fait que la tâche "énigme" n'exige pas la traduction d'une information originalement non langagière (par exemple iconographique) sous une forme langagière (l'information est déjà textuelle), et qu'elle peut au final être résolue par chacun des participants de manière privée (après obtention des indices de l'autre), pourrait expliquer que la communication 
s'accompagne de moins de demandes de clarification ou plus généralement de rétroactions visant à préciser le contenu sémantique de l'interlocuteur.

De ces différentes remarques, on peut inférer l'hypothèse suivante, qu'une tâche dont la résolution exige : 1) une collaboration réelle des participants, 2) une forte précision descriptive, et 3) la "traduction" d'une information non langagière en une information langagière pour sa transmissibilité, favorise la génération de séquences d'interaction langagière susceptibles d'amorcer des processus d'acquisition ou plus largement de restructuration de l'interlangue sur lesquels repose l'apprentissage. Nous rejoignons ici en partie la description des caractéristiques de la tâche faite par Chapelle (2001), Ellis (2003) et Narcy-Combes (2005).

Par ailleurs, la dimension collaborative dépend elle-même dans une certaine mesure de la dimension motivationnelle évoquée ci-avant: si on peut supposer qu'une tâche dont la résolution exige une véritable collaboration de la part des membres du binôme les engagera de façon plus immersive dans l'interaction, et pourra donner lieu à plus d'interactions négociées, seule une tâche attractive (en particulier une tâche où il y a une forme d'enjeu) courtcircuitera de possibles effets de lassitude conduisant à l'arrêt de la tâche avant sa résolution. Un bon dosage de la difficulté de la tâche s'avère sur ce point un élément décisif.

On peut finalement faire les propositions suivantes sur les caractères que doit posséder une tâche pour générer des rétroactions correctives et plus généralement de la négociation :

- sa résolution doit exiger une réelle collaboration de la part des membres du binôme (la tâche ne doit pas pouvoir être résolue seule) ;

- les informations à partager pour la résolution de la tâche ne doivent pas être immédiatement transmissibles, mais un jeu de va-et-vient doit au contraire s'installer;

- la résolution de la tâche doit exiger une grande précision descriptive (ce qui peut favoriser la production de rétroactions correctives visant le contenu sémantique, et plus généralement la génération de demandes de clarification).

On fera remarquer pour finir que l'absence de post-test après les tâches (faute de pouvoir exiger des participants un investissement trop important) ne nous permet pas de déterminer si des appropriations ont ou non été réalisées suite aux communications et aux négociations qui 
les ont jalonnées. Comme l'a fait remarquer Narcy-Combes (2005), il peut être inadapté de valider une tâche "communicative" par un pré-test et un post-test qui mesurent la compétence linguistique. En revanche, ces tests peuvent aider à déterminer si une tâche (ou plus généralement une activité d'apprentissage) est plus efficace qu'une autre. Pour vérifier s'il y a ou non appropriation, des post-tests répétés à des intervalles de temps réguliers sont absolument nécessaires (voir les études de Klein, 1989; Véronique, 1992, 2007 ; Carroll, 1984 ; Long et Robinson, 1998). En effet, si quelques recherches ont indiqué que la capacité des apprenants à modifier leur output dans la réponse à la rétroaction suggérait qu'ils auraient utilisé dans quelque mesure cette rétroaction (Mackey, Oliver et Leeman, 2003 : 48), prise isolément, l'incorporation (uptake) immédiate à la rétroaction corrective (répétition de la réparation de la part de l'apprenant) n'est pas un indicateur suffisamment fiable des effets à long terme de la rétroaction négative, c'est-à-dire de l'acquisition (Gass, 1997 ; Mackey \& Philip, 1998).

\section{Références}

\section{Bibliographie}

Blake, R. (2000). "Computed Mediated Communication: a Window on L2 Spanish Interlanguage". Language Learning and Technology, vol. 4, n 1. pp. 120-136.

Braidi, S.M. (2002). "Reexamining the Role of Recasts in NS / NNS Interactions". Language Learning, vol. 52, $\mathrm{n}^{\circ} 1$. pp. 1-42.

Carroll, M. (1984). Cyclical Learning Processes in Second Language Production. Frankfurt am M. : Lang.

Chun, D.M. (1994). "Using Computer Networking to Facilitate the Acquisition of Intercative Competence". System, vol. 22, $\mathrm{n}^{\circ} 1$. pp. 17-31.

Ellis, R. (2003). Task-based Language Learning and Teaching. Oxford : OUP.

Gass, S. \& Varonis, E. (1985). "Task Variation and Nonnative / Nonnative Negotiation of Meaning". In Gass, S. \& Madden, C. (dir.). Input in Second Language Acquisition. pp. 149161. Rowley, M.A. : Newbury House Publishers. 
Gass, S.M. (1988). "Second Language Vocabulary Acquisition". Annual Review of Applied Linguistics, $\mathrm{n}^{\circ}$ 9. pp. 92-106.

Gass, S.M. (1997). "Input, Interaction, and Second Language Learner". Mahwah, NJ : Erlbaum.

Gass, S.M. (1998). "Apples and Oranges: Or, Why Apples are not Oranges and Don't Need to Be". Modern Language Journal, vol. 82. pp. 83-90.

Gass, S., Mackey, A. \& Ross-Feldman, L. (2005). "Task-Based Interactions in Classroom and Laboratory Settings". Language Learning, vol. 55, n 4. pp. 575-611.

Kazeroni, A. (2004). "La construction d'une tâche d'apprentissage d'une langue étrangère dans des environnements informatiques". Les Études de Linguistique Appliquée. Usages des nouvelles technologies dans l'enseignement des langues étrangères, $\mathrm{n}^{\circ} 134$. pp.159-171.

Kern, R. (1995). "Restructuring Classroom Interaction with Networked Computers: Effects on Quantity and Characteristics of Language Production". Modern Language Journal, vol. 79. pp. 457-476.

Klein, W. (1989). L'acquisition de langue étrangère. Paris : Armand Colin.

Lightbown, P.M. \& Spada, N. (1999). "How Languages are Learned". Oxford : OUP.

Long, M. (1996). "The Role of the Linguistic Environment in Second Language Acquisition". In Ritchie, W. \& Bhatia, T. (dir.). Handbook of Second Language Acquisition. pp. 413-468. New York : Academic Press.

Long, M. \& Robinson, P. (1998). "Focus on Form: Theory, Research and Practice". In Doughty, C. \& Williams, J. (dir.). Focus on Form in Classroom Second Language Acquisition. pp. 15-41. New York : Cambridge University Press.

Long, M.H. (2005). "The Recast. Plenary Conference". UNTELE 2005, Compiègne.

Lyster, R. \& Ranta, L. (1997). "Corrective Feedback and Learner Uptake ". Studies in Second Language Acquisition, n ${ }^{\circ}$ 19. pp. 37-36. 
Mackey, A. \& Philip, J. (1998). "Conversational Interaction and Second Language Development: Recasts, Responses, and Red Herrings?". Modern Language Journal, n 82. pp.338-356.

Mackey, A. \& Gass, S.M. (2005). "Second Language Research: Methodology and Design". Mahwah, NJ : Erlbaum.

Mackey, A., Oliver, R. \& Leeman, J. (2003). "Interactional Input and the Incorporation of Feedback: an Exploration of NS-NNS Adult and Child Dyads". Language Learning, vol. $\mathrm{n}^{\circ}$ 53. pp. 35-66.

Narcy-Combes, J-P. (2005). Didactique des langues et TIC: vers une recherche-action responsable. Paris : Ophrys.

Negretti, R. (1999). "Web-based Activities and SLA: a Conversation Analysis Research Approach". Language Learning and Technology, vol. 3. pp. 75-87.

Pelletieri, J. (2000). "Negotiation in Cyberspace - the Role of Chatting in the Development of Grammatical Competence". In Warschauer, M. \& Kern R. (dir.). Network-based LT: Concepts and Practice. Cambridge : CUP.

Pica, T. (1994). "Research on Negotiation: What Does it Reveal about Second Language Learning Conditions, Processes, and Outcomes ?". Language Learning, vol.44. pp. 493-527.

Prabhu, N.S. (1987). Second Language Pedagogy. Oxford : OUP.

Tarone, E \& Franck A. Morris (2003). "Impact of Classroom Dynamics on the Effectiveness of Recasts in SLA". Language Learning, ${ }^{\circ}$ 53. pp.325-368.

Tatawy, M.E. (2002). "Corrective Feedback in Second Language Acquisition". Working Papers in TESOL and Applied Linguistics, Teachers College, Columbia University, vol. 2, $\mathrm{n}^{\circ}$ 2.

Tudini, V. (2003). "Using Native Speakers in Chat". Language Learning and Technology, n7. pp. 141-159.

Véronique, D. (1992). "Recherches sur l'acquisition des langues secondes : un état des lieux et quelques perspectives". Aile, $\mathrm{n}^{\circ} 1$. Encrages. pp. 5-37. 
Véronique, D. (2007). "L’apport des recherches sur l'acquisition des langues étrangères". Français dans le monde. pp.96-106.

Warschauer, M. (1996). "Comparing Face-to-face and Electronic Communication in the Second Language Classroom". CALICO Journal, vol. 13. pp. 7-25.

\section{À propos de l'auteure}

Elena Coşereanu est doctorante en Didactique du FLE au laboratoire COSTECH à l'Université de Technologie de Compiègne.

Courriel : elena.cosereanu@utc.fr.

Adresse : Centre Pierre Guillaumat, Costech, BP 60319, 60203 Compiègne Cedex, France. 